\title{
Spatial Analysis of Colorectal Cancer in Iran
}

\author{
Reza Pakzad ${ }^{1,2}$, Asieh Moudi ${ }^{3}$, Zahra Pournamdar ${ }^{4}$, Iraj Pakzad ${ }^{5,6}$, Abdollah \\ Mohammadian-Hafshejani ${ }^{7}$, Zohre Momenimovahed ${ }^{8}$, Hamid Salehiniya ${ }^{2,9 *}$, \\ Farhad Towhidi $^{10}$, Behnam Reza Makhsosi ${ }^{10}$
}

\begin{abstract}
Colorectal cancer is one of the most common cancers. Due to demographic changes, it is predicted that the incidence of this cancer will increase. Variations of its incidence rate among geographical areas are due to various contributing factors. Since there have been a lack of studies on this topic in our country, the present assessment of spatial patterns of colorectal cancer incidence in Iran was performed. In this ecological study, the new cases of colon cancer were extracted from Cancer Registry Center report of the Health Deputy of Iran in 2009. The reported incidences of the disease were standardized on the basis of the World Health Organization population and the direct method. Then the data were inserted into the GIS software, and finally, using the analysis of hot spots (Getis-Ord Gi) high-risk areas were drawn. Provinces that are higher or lower than the national average (1.9 SD) were considered hot spots or cold spots, significant at the level of 0.05 . A total of 6,210 cases of colorectal cancer were registered in Iran in 2009, of which 3,727 were in men and 2,783 in women (age-standardized rates of 11.3 and 10.9 per 100,000 population, respectively). The results showed that in central and northern Iran including Isfahan, Qom, Tehran, Qazvin and Mazandaran significant hot spots in men were present $(\mathrm{p}<0.05)$. In women also we have high incidence in northern and central states: Mazandaran province $(p<0.01)$ and the province of Tehran $(p<0.05)$ had higher incidences than the national average and were apparent as significant hot spots. Analysis of the spatial distribution of colorectal cancer showed significant differences between different areas pointing to the necessity for further epidemiological studies into the etiology and early detection.
\end{abstract}

Keywords: Colorectal cancer - incidence - geographical variation - Iran

Asian Pac J Cancer Prev, 17, Cancer Control in Western Asia Special Issue, 53-57

\section{Introduction}

Cancer is a major public health problem in Iran and many other parts of the world (Organization, 2011; Pakzad et al., 2015a; Razi et al., 2015). In 2012, about 14.1 million new cases of cancer were reported in the world and 8.2 million of them led to death. However, it is predicted That the number of new cases in 2030 will reach to 21.7 million people and the number of deaths due to cancer to 13 million and these numbers indicate an increase in the burden of disease in the future (Globocan, 2012; Pakzad et al., 2015b).

One of the world's most common cancers is colorectal cancer. With 400 thousand deaths annually in the world, it is allocated as the third leading cause of cancer death (Parkin, 2001; Li and Gu, 2005). The results of the global distribution of colorectal cancer shows that the incidence and prevalence of colorectal cancer are very different in the world. In some areas, this difference reaches to 25 times
(Schottenfeld and Winawer, 1996; Parkin et al., 2001; Siegel et al., 2014). The highest incidence of colorectal cancer is in North America, Western Europe, Australia and Japan. This amount is estimated of about 30 to 50 per 100,000 people (Schottenfeld and Winawer, 1996; Siegel et al., 2014). But moving to the African, Mediterranean and Asian countries, the incidence of colorectal cancer reduces and it reaches to 3-7 per 100,000 people (Muir et al., 1987; Ansari et al., 2006; Siegel et al., 2014; Torre et al., 2015). Various studies in developed and Western countries have showed that only $2-8 \%$ of colorectal cases happen in under 40 year old people (Bülow, 1980; Mitry et al., 2001). While this rate reaches to around 15 to 35 per cent in the Middle East and Asia (Sung et al., 2005; Ansari et al., 2006; Siegel et al., 2014).

The difference in distribution of colorectal cancer in the world can be attributed to two Factors: A Real difference in the incidence of colorectal cancer that can be caused by differences in variety of risk factors that

${ }^{2}$ Department of Epidemiology and Biostatistics, School of Public Health, 'Students' Scientific Research Center, Tehran University of Medical Sciences, ${ }^{9}$ Minimally Invasive Surgery Research Center, Iran University of Medical Sciences, Tehran, ${ }^{3}$ Birjand University of Medical Sciences, Birjand, ${ }^{4}$ pregnancy health research center, Zahedan University of Health Sciences, Zahedan, Department of Microbiology, Medical School, ${ }^{6}$ Clinical Microbiology Research center, Ilam University of Medical Sciences, Ilam, ${ }^{7}$ Dept. of Social Medicine, School of Medicine, Rafsanjan University of Medical Sciences, Rafsanjan, ${ }^{8}$ Qom University of Medical Sciences, Qom, ${ }^{10}$ Imam Reza Hospital, Kermanshah University of Medical Sciences, Kermanshah, Iran.*For correspondence: alesaleh70@yahoo.com 
the most important of which is changes in lifestyle and personal habits. B. Pseudo difference in the incidence of disease which could be due to differences in methods of data collection, differences in access to health services, and diagnostic and screening facilities (Mousavi et al., 2009; Edwards et al., 2010). However, a significant part of this difference can be attributed to lifestyle and personal habits (Mousavi et al., 2009; Pourfarzi et al., 2012; Almasi and Salehiniya, 2015).

Also, in Iran colorectal cancer is among the most common cancers it comprises the third level in men, fourth level in women (Alireza et al., 2005) and fifth degree totally (Mousavi et al., 2009). This cancer was among the cancers with low incidence before, but due to the lifestyle changes in Iran, its incidence is increasing (Mousavi et al., 2009; Radmard, 2010). Its incidence is 7 and 8 per 100000 people in men and women respectively (Ansari et al., 2006). The colorectal cancer incidence in Iran is similar to its incidence in the Middle East countries but it is lower than Western ones (Radmard, 2010).

There are a few studies about the colorectal cancer distribution in Iran. However, the results of these studies indicate a difference in the incidence of colorectal cancer in Iran (Mousavi et al., 2009). Almasi in his study showed that the incidence of colorectal cancer in northern and central provinces is higher than other regions (Almasi and Salehiniya, 2015). Esmarian also noted high incidence of colorectal cancer in Northern areas of the country (Asmarian et al., 2013).

Reviewing the geographical distribution of colorectal cancer can lead to better understanding of its incidence pattern and impact of environmental factors. One of the tools that can measure the distribution of disease, and determine high risk areas and the impact of environmental factors is Geographical Information System. There are several studies on the usage of this system in the same disease (Poulstrup and Hansen, 2004; Rushton et al., 2004; Mousavi et al., 2009; Yomralioglu et al., 2009). Due to the fact that the knowledge about the spatial characteristics of the disease can help in prioritizing and providing appropriate services for health in high-risk areas and also due to lack of similar studies which investigate spatial analysis on colorectal cancer in Iran, we decided to investigate the spatial distribution of colorectal cancer in Iran.

\section{Material and Methods}

\section{The study area}

Iran is a country in the Middle-East with the area of $1,648,195$ square-kilometers, it is the 18 th largest country in the world and has a population of over 75 million (2011) (Keller et al., 2013). Iran has a Variety of environmental conditions in terms of length and breadth of geography, topographic diversity, and a range of altitude up to 5,671 meters above sea level. The Iranian climate varies from arid to subtropical. Iran is bordered by Armenia, Turkmenistan, and Azerbaijan to the North, (as well as by Russia and Kazakhstan via a water border in the Caspian Sea); Afghanistan and Pakistan to the East; the Persian Gulf and Gulf of Oman to the south; Iraq to the east; and
Table 1. Standardized Incidence Rate of Colorectal Cancer in Iranian Provinces Base on Sex

\begin{tabular}{lll}
\hline Province & Female & Male \\
\hline Ardebil & 9.6 & 11.8 \\
Esfahan & 12.0 & 13.3 \\
Ilam & 7.2 & 6.5 \\
East- Azarbaijan & 11.9 & 14.0 \\
West-Azerbaijan & 8.5 & 8.1 \\
Bushehr & 7.1 & 5.0 \\
Tehran & 17.6 & 16.4 \\
Chaharmahal-e Bakhtiari & 6.1 & 7.1 \\
South Khorasan & 6.9 & 8.5 \\
Khorasan-e Razavi & 11.9 & 11.8 \\
North Khorasan & 2.3 & 5.6 \\
Khuzestan & 9.9 & 10.5 \\
Zanjan & 5.0 & 7.5 \\
Semnan & 14.4 & 16.1 \\
Sistan and Baluchestan & 3.0 & 2.5 \\
Fars & 10 & 8.7 \\
Qazvin & 11.2 & 8.3 \\
Qom & 5.8 & 7.0 \\
Kurdistan & 8.6 & 8.0 \\
Kerman & 8.6 & 7.1 \\
Kermanshah & 9.7 & 10.9 \\
Kohgiluyeh va Boier ahmad & 3.1 & 7.5 \\
Golestan & 10.0 & 8.1 \\
Guilan & 14.3 & 17.2 \\
Lurestan & 6.7 & 7.6 \\
Mazandaran & 12.6 & 12.4 \\
Markazi & 12.5 & 12.4 \\
Hormozgan & 4.0 & 2.6 \\
Hamedan & 9.8 \\
Yazd & 12.0 \\
Total & 11.3 \\
\hline
\end{tabular}

Turkey to the northwest (Mostafavi et al., 2013) Iran is currently divided into 31 provinces.

Study design and data collection

This is an ecological study in Iran. The data were collected from the report of health deputy of Cancer Registry Center which is based on Iran ministry of

$$
G_{i}^{*}=\frac{\sum_{j=1}^{n} w_{i, j} x_{j}-\bar{X} \sum_{j=1}^{n} w_{i, j}}{S \sqrt{\frac{\left[n \sum_{j=1}^{n} w_{i, j}^{2}-\left(\sum_{j=1}^{n} w_{i, j}\right)^{2}\right]}{n-1}}}
$$

Figure 3. Getis-Ord Gi* (Spatial Statistics) - where $\mathrm{Xi}$ is the Attribute Value for Feature $\mathrm{j}$, wij is the Spatial Weight between Feature $\mathrm{i}$ and $\mathrm{j}, \mathrm{n}$ is Equal to the Total Number of Features. 


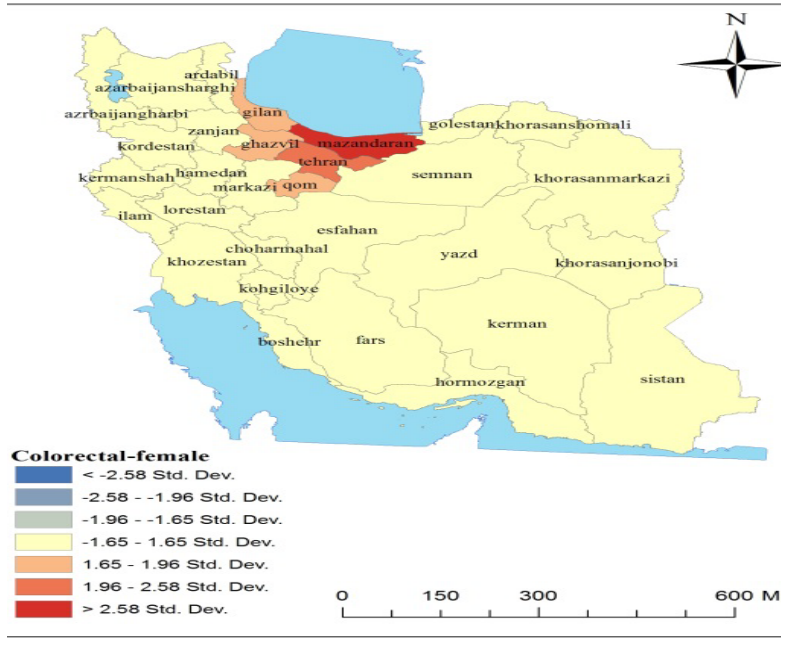

Figure 1. Hot Spots of Colorectal Cancer in Females in 2009

health guideline (Goya, 2007). Data were collected retrospectively reviewing all new colon cancer patients in cancer registry center report of Health deputy of Iran in 2009. Colon cancer was defined as icd-oc16. Age-standardized rates of cancer incidence were calculated by the direct standardization method, using the world standard population as a reference (Rahimi and Heidari, 2012).

After data collection, they were inserted into Excel datasheets. Using Excel was justified by the faster computational capabilities and the ability to convert and store data in tables which can be linked to geographical maps in ArcGIS software, as well as being an interactive environment for checking numbers simultaneously when inserting data.

\section{Spatial analysis by GIS}

The most recent updated electronic map of Iran and its provinces was used. To link data tables to the map, a so-called ID field was created in Excel tables reporting the incidence of colon cancer. The code entered corresponded to the code of a province in the map data tables. The tables and map were then linked by a common field.

Identifying disease hotspots was done using Getis-Ord Gi* (Spatial Statistics) (Figure 1). An incidence hotspot was defined as a province which not only has a high incidence of disease itself but also has a neighboring province with a high incidence. In other words, the hotspot analysis shows that provinces with high incidence of disease become a focus of disease if there is high incidence in surrounding provinces as well. To the contrary, it can be said that 'Cold spots' are provinces with not only low incidence but also with low-incidence neighbors. Provinces that are higher or lower than the national average (1.9 SD) were considered Hot spots or Cold spots as were significant at the level of $0.05 \%$.

\section{Results}

In 2009 , a total of 6,210 cases of colorectal cancer

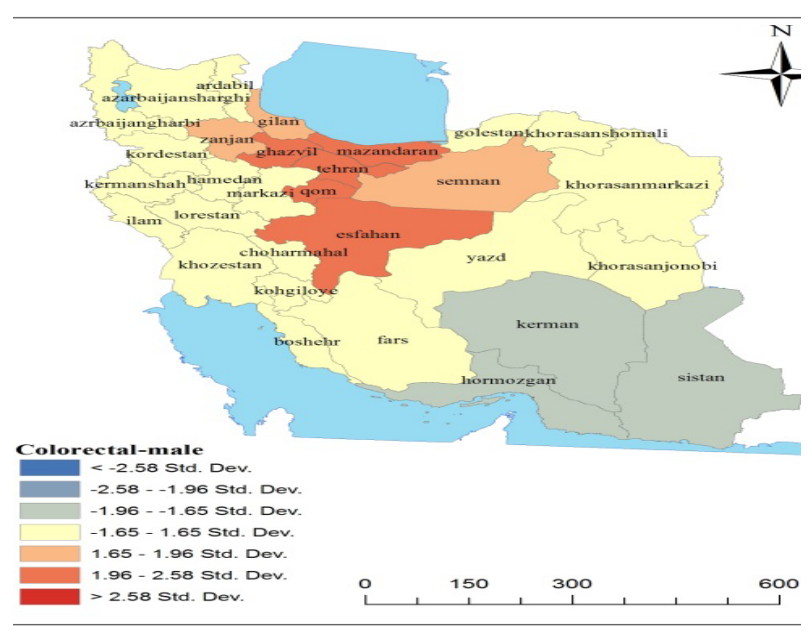

Figure 2. Hot Spots of Colorectal Cancer in Males in 2009

occurred in Iran of which 2,783 were women and 3,727 were men. The annual incidence of colorectal cancer was 11.3 per hundred thousand people in men and 10.9 per hundred thousand people in women. Our study showed that the highest incidence was in women of Tehran province with 17.6 per hundred thousand people. Then Semnan with 14.4, Gilan with 14.3, Mazandaran with 12.6 and central provinces with 12.5 per hundred thousand people had the most incidence. Also the highest incidence of colorectal cancer in men was in Gilan province with 17.2 per hundred thousand people. Then the provinces of Tehran with 16.5, Semnan with 16.1, East Azerbaijan with 14 and Esfahan with 13.3 per hundred thousand people had the highest incidence.

Also the results showed that the distribution of areas with a high incidence of the disease was in central and Northern provinces of the country. Figures 2 and 3 are for the analysis of colorectal cancer hot spots in men and women. Figure 2, which is related to colorectal cancer in women, shows that hot spots of the disease are in central and Northern provinces of the country. Tehran $(\mathrm{p}<0.05)$ and Mazandaran $(\mathrm{p}<0.01)$ provinces are classified as significant hot and too hot spots respectively. Although the provinces of Qom, Qazvin and Rasht had also a higher incidence than the national average, this difference was not significant.

Figure 3, which is related to colorectal cancer in men, shows that again the northern and central provinces of the country have high incidence Isfahan, Qom, Tehran, Qazvin And Mazandaran had higher incidence than the national average and were known as significant hot spots $(\mathrm{p}<0.05)$. The provinces of Semnan, Guilan and Zanjan had higher incidence than the national average, but this difference was not significant. Also the provinces of Sistan-Baluchestan, Hormozgan and Kerman were identified as low risk areas for colorectal cancer but their difference was not significant at the 0.05 level.

\section{Discussion}

Our results showed that colorectal cancer has a higher incidence in northern and central provinces of country than 
the rest of areas most of the hot spots of the disease were in these regions. In contrast, cancer incidence in south and southeast of the country is lower than other provinces. Paying attention to the fact that the majority of the population is concentrated in these areas (Keller et al., 2013), giving priority to these regions in order to provide cancer control programs and lifestyle changes has great importance (Pakzad et al., 2015a).

Almasi in his study showed that the colorectal cancer has the highest rate in Northern provinces, and in East and Southeast of the country this rate is low (Almasi and Salehiniya, 2015). Esmarian et al with spatial analysis showed that the highest incidence of the disease was in northern and central provinces such as Mazandaran, Gilan, Isfahan and Tehran. They also showed that the Country's southeastern provinces such as Sistan-Baluchestan, Kerman and Bushehr have low incidence (Asmarian et al., 2013). The results of these studies and our findings are consistent. It seems that differences in lifestyle and eating habits, tending to low-fiber high-fat diet, rising levels of obesity and fast food consumption (Edwards et al., 2010) have prepared a fit condition for the citizens of these areas to suffer from the disease. Tendency to mechanical life and low physical activity are also other risk factors.

Although the difference in colorectal cancer incidence in Iran can be because of the prevalence of risk factors, it should be noted that most of the southern provinces of the country are among deprived areas and low socio-economic classes (Hosseinpoor et al., 2006; Donyavi et al., 2011; Almasi et al., 2015). Because Of this reason the difference can be attributed to lack of access to diagnostic and health services, lack of comprehensive cancer program and lack of reporting or no reporting (Mousavi et al., 2009; Pourhoseingholi et al., 2009; Edwards et al., 2010; Keyghobadi et al., 2015). In contrast, the northern and central provinces are among high socio-economic class provinces. It has been shown that with enhancement of socio-economic class, lifestyle changes and most people take the Western lifestyle. This pattern that comes with lifestyle changes and changing the traditional diet to low fiber and high in calories is associated with increased colorectal cancer (Ansari et al., 2006).

Among other findings of our study was the priority of cancer incidence in men than women. The disease incidence was 11.31 per hundred thousand people in men and 10.89 per hundred thousand people in women. Ansari found the age standardized incidence rate for colorectal cancer 8.2 and 7.1 per hundred thousand people for men and women respectively (Ansari et al., 2006). Almasi also reported the sex ratio of 1.33 that shows the cancer incidence excellence in men than women (Almasi and Salehiniya, 2015). This difference in incidence can be attributed to men's higher exposure to risk factors including foods high in fat, low physical activity and smoking. Pourfarzi observed a significant relationship due to the protective effect of fruit and vegetable intake and confirmed positive the increasing role of carbohydrate intake, history of smoking and a family history (Pourfarzi et al., 2012). However, the incidence of colorectal cancer in Iranian population is similar to the population of Middle East and is much less than cancer in developed and Western countries (Radmard, 2010).

Although most cancers are preventable in theory and they can be diagnosed with screening programs, these programs for colorectal cancer are low in Iran and other countries. And it may be due to high cost and resistance by physicians, patients, and the healthcare system (Mousavi et al., 2009). However, in Iran it is recommended that people with a family history of colorectal cancer use diagnostic procedures, including colonoscopy and fecal occult blood test in middle ages for early detection of cancer (Radmard, 2010).

In this study, we tried using geographic information systems to identify any variation in the distribution of the disease. In this study the Hot Spots determination was done with Getis-Ord Gi* (Spatial Statistics). Although high-risk areas in this study indicate their importance in incidence of colorectal cancer, it should be noted that several factors may be involved. Perhaps the high incidence of risk factors in Hot Spots explain these differences, but other factors such as incomplete data registration, lack of a full report and bad data classification can also be among effective factors in differences of the disease incidence in the country. For this reason, when using national data to examine spatial conditions, one should also pay attention to these fators.

Colorectal cancer is now among the most common cancers in Iran. Due to the high incidence of colorectal cancer in central and Northern provinces, giving priority to these provinces to provide preventive and curative services can be helpful in reducing the incidence of disease.

\section{References}

Alireza S, Mehdi N, Ali M, et al (2005). Cancer occurrence in Iran in 2002, an international perspective. Asian Pac J Cancer Prev, 6, 359.

Almasi SZ, Salehiniya H (2015). Trends in Colorectal Cancer Incidence in Iran. J Mazandaran Univ Med Sci, 24, 389-94.

Almasi Z, Rafiemanesh H, Salehiniya H (2015). Epidemiology characteristics and trends of incidence and morphology of stomach cancer in Iran. Asian Pac J Cancer Prev, 16, 2757-61.

Ansari R, Mahdavinia M, Sadjadi A, et al (2006). Incidence and age distribution of colorectal cancer in Iran: results of a population-based cancer registry. Cancer Lett, 240, 143-7.

Asmarian NS, Kavousi A, Salehi M (2013). Mapping of large intestine cancer incidence rate using area-to-area Poisson Kriging in Iran from 2003 to 2007. Razi J Med Sci, 20, 10-7.

Bülow S (1980). Colorectal cancer in patients less than 40 years of age in Denmark, 1943-1967. Dis Colon Rectum, 23, 327-36.

Donyavi T, Naieni KH, Nedjat S, et al (2011). Socioeconomic status and mortality after acute myocardial infarction: a study from Iran. Int J Equity Health, 10, 9-15.

Edwards BK, Ward E, Kohler BA, et al (2010). Annual report to the nation on the status of cancer, 1975-2006, featuring colorectal cancer trends and impact of interventions (risk factors, screening, and treatment) to reduce future rates. Cancer, 116, 544-73.

Goya M (2007). Iranian Annual Cancer Registration Report 2005/2006. Ministry of Health and Medical Education, Health Deputy. Center for Disease Control and Prevention .In Persian. 
Hosseinpoor AR, Van Doorslaer E, Speybroeck N, et al (2006). Decomposing socioeconomic inequality in infant mortality in Iran. Int. J. Epidemiol, 35, 1211-9.

Keller DI, Bizzini M, Feddermann N, et al (2013). FIFA Women's World Cup 2011: pre-competition medical assessment of female referees and assistant referees. $\mathrm{Br} J$ Sports Med, 47, 179-81.

Keyghobadi N, Rafiemanesh H, Mohammadian-Hafshejani A, et al (2015). Epidemiology and trend of cancers in the province of Kerman: southeast of Iran. Asian Pac J Cancer Prev, 16, 1409-13.

Li M, Gu J (2005). Changing patterns of colorectal cancer in China over a period of 20 years. World J Gastroenterol, 11, 4685-8.

Mitry E, Benhamiche AM, Jouve JL, et al (2001). Colorectal adenocarcinoma in patients under 45 years of age: comparison with older patients in a well-defined French population. Dis Colon Rectum, 44, 380-7.

Mostafavi E, Haghdoost A, Khakifirouz S, et al (2013). Spatial analysis of Crimean Congo hemorrhagic fever in Iran. $\mathrm{Am}$ J Trop Med Hyg, 89, 1135-41.

Mousavi SM, Gouya MM, Ramazani R, et al (2009). Cancer incidence and mortality in Iran. Ann. Oncol, 20, 556-63.

Muir C, Waterhouse J, Mack T, et al (1987). Cancer incidence in five continents: International agency for research on cancer. IRAC Scientific Publication, 88.

Pakzad R, Mohammadian-Hafshejani A, Mohammadian M, et al (2015a). Incidence and Mortality of Bladder Cancer and their Relationship with Development in Asia. Asian Pac J Cancer Prev, 16, 7365-74.

Pakzad R, Soltani S, Salehiniya H (2015b). Epidemiology and trend in skin cancer mortality in Iran. J Res Med Sci, 20, 921.

Parkin DM (2001). Global cancer statistics in the year 2000. Lancet Oncol, 2, 533-43.

Parkin DM, Bray F, Devesa S (2001). Cancer burden in the year 2000. The global picture. Eur J Cancer, 37, 4-66.

Poulstrup A, Hansen H (2004). Use of GIS and exposure modeling as tools in a study of cancer incidence in a population exposed to airborne dioxin. Environ Health Perspect, 112, 1032-6.

Pourfarzi F, Yazdanbod A, Daneshvar R, et al (2012). Evaluation of effective factors in incidence of colorectal cancer. $J$ Ardabil Univ Med Sci, 12, 56-64.

Pourhoseingholi MA, Faghihzadeh S, Hajizadeh E, et al (2009). Bayesian estimation of colorectal cancer mortality in the presence of misclassification in Iran. Asian Pac J Cancer Prev, 10, 691-4.

Radmard AR (2010). Five common cancers in Iran. Arch Iran Med, 13, 143-6.

Rahimi F, Heidari M(2012). Time trend analysis of stomach cancer incidence in the west of Iran. J Mazandaran Univ Med Sci, 19, 61-5.

Razi S, Rafiemanesh H, Ghoncheh M, et al (2015). Changing trends of types of skin cancer in Iran. Asian Pac $J$ Cancer Prev, 16, 4955-8.

Rushton G, Peleg I, Banerjee A, et al (2004). Analyzing geographic patterns of disease incidence: rates of late-stage colorectal cancer in Iowa. $J$ Med Syst, 28, 223-36.

Siegel R, DeSantis C, Jemal A (2014). Colorectal cancer statistics, 2014. CA Cancer J Clin, 64, 104-17.

Sung JJ, Lau JY, Goh K, et al (2005). Increasing incidence of colorectal cancer in Asia: implications for screening. Lancet Oncol, 6, 871-6.

Torre LA, Bray F, Siegel RL, et al (2015). Global cancer statistics, 2012. CA Cancer J Clin, 65, 87-108.

Yomralioglu T, Colak EH, Aydinoglu AC (2009). Geo-relationship
DOI:http://dx.doi.org/10.7314/APJCP.2016.17.S3.53 Spatial Analysis of Colorectal Cancer in Iran

between cancer cases and the environment by GIS: A case study of Trabzon in Turkey. Int J Environ Res Public Health, 6, 3190-204. 